\title{
SOLUTION OF A MODEL FOR TWO-DIMENSIONAL INTRADIFFUSION
}

\author{
G. Erochenkova \\ Centre de Physique Théorique (Unité Propre de Recherche 7061), \\ CNRS-Luminy, Case 907 F-13288 Marseille, Cedex 9, France
}

(Received February 20, 2001; received in final form November 16, 2001)

\begin{abstract}
A system of partial differential equations corresponding to two-dimensional intradiffusion (Wicke) model for a weakly nonlinear isotherm of sorption is solved by reducing it to the Burgers' equation. Some limiting cases interesing from the physical point of view are considered in details.

Key words: diffusion, dynamics of sorption, packed-bed.
\end{abstract}

PACS number(s): 02.30.Jr, 68.43.Jk

\section{INTRODUCTION}

The microscopic physical description of the sorption/desorption phenomenon is a rather complicated problem. It is especially the case, when this phenomenon is connected with diffusion into a sorption medium. In this case one considers a flow of inactive liquid or gas (a mobile phase) through a bed homogeneously packed by small solid spheres covered by sorbing film (a sorption medium) with a packing characteristic porosity $\varepsilon=$ void volume . The aim of this note is to study dynamics of sorption of an active marker introduced into a mobile phase. The process of permeability of this marker through a sorption medium is based essentially on a physical picture of multiply repeated sorption and desorption acts.

The mathematical description of dynamics of sorption has to take into account the following basic peculiarities of this complicated phenomenon: balance of an active marker in process of displacement into a sorption medium, kinetic and static properties of this marker, hydrodynamics of this process, dependence between thermodynamic condition parameters of a sorption medium, balance of heat and heat transfer in the process of dynamics of sorption in a mobile phase, etc. Because of complexity of this physical picture the theory develops via formulation of different simplifications and approximations. Usually they are the following (see, e. g., [1-4] and references therein):

- dynamics of sorption is isothermal;

- a mobile phase is incompressible and a concentration of an active marker is so small that variation of a density of sorbing marker can be neglected;

- a flow of a mobile phase is either one dimensional, or one considers an axial-symmetric problem;

- one can neglect the non-homogeneity of a sorption medium.

The main problem of the theory of dynamics of sorption now can be formulated as follows: to find the time (or space) distribution of concentration of sorbing marker for each length $x$ (or for each moment of time $t$ ), if the initial concentration of the marker into mobile phase and character of interaction between marker and a sorption medium are known.

Usually a concentration of the sorbing marker into the mobile phase and a concentration of the sorbed marker into the sorption medium are averaged over the crosssection, thus they are functions of one variable $x$ ([24]). But more precise consideration should take into account a variation of concentration of sorbed marker into a sorption medium along the radius of packing particles. Wicke [5] proposed a two-dimension intradiffusion model (the mathematical formulation is due to Tunitskij et al. [6]) where concentration of the sorbed marker results from diffusion of the sorbing marker into a layer of a sorption medium (see Fig. 1).

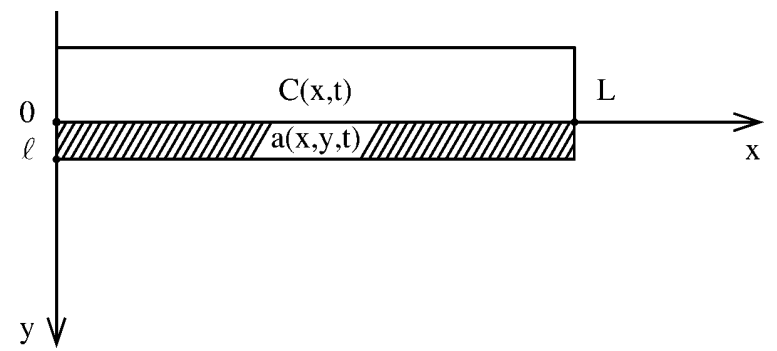

Fig. 1. Diffusion of a sorbing marker $c(x, t)$ into a layer of a sorption medium with thickness $l$.

In the present paper it is shown that the twodimension intradiffusion Wicke model can be reduced to a partial differential equation for the concentration of the sorbing marker into the mobile phase. Moreover, this equation is solved explicitly for a weakly nonlinear isotherm of sorption.

\section{THE MATHEMATICAL SET UP}

The system of the partial differential equations corresponding to Wicke model has the form [6] (see Fig. 1):

$$
\frac{\partial a}{\partial t}=\mathcal{D}_{f} \frac{\partial^{2} a}{\partial y^{2}},
$$




\section{G. EROCHENKOVA}

$\left.\frac{s \mathcal{D}_{f}}{\varepsilon} \frac{\partial a}{\partial y}\right|_{y=0}=\frac{\partial c}{\partial t}+u \frac{\partial c}{\partial x}$,

$\left.\frac{\partial a}{\partial y}\right|_{y=l}=0$,

$\left.c(x, t)\right|_{t=0}=f(x)=\left\{\begin{array}{ll}c_{0}, & 0 \leq x \leq x_{0} \\ 0, & x>x_{0}\end{array}\right.$,

$\left.a(x, y, t)\right|_{t=0}= \begin{cases}\left.F(c(x, t))\right|_{t=0}=\psi(x), & y=0 \\ 0, & y>0\end{cases}$

Here $a=a(x, y, t)$ is the concentration of the sorbed marker into the sorption medium, and $a(x, y=0, t)=$ $F(c(x, t))$ is the equation of the isotherm of sorption; $c=c(x, t)$ is the concentration of the sorbing marker into the mobile phase; $y$ is the coordinate inside the film of the sorption medium of thickness $l ; \mathcal{D}_{f}$ is the diffusion coefficient into the sorption medium; $u$ is the linear flow velocity which is a constant in the cross-section and along the column length; $s$ is the specific area of the surface boundary between a mobile phase and a sorption medium (per unit bend length and per unit bend crosssection); $\varepsilon$ is the porosity.

Notice that the Wicke model describes in fact a twodimensional diffusion (into directions $y$ and $x$ ) corresponding to the sorbed $a$ and the sorbing $c$ markers related by some isotherm equation.

\section{EVOLUTION EQUATION FOR THE MODEL (1)-(5)}

Taking into account (5) we can represent the solution $a(x, y, t)$ in the form:

$$
a(x, y, t)=\Theta(x, y, t)+F(c(x, t)) \text {. }
$$

Then by (1) the new function $\Theta=\Theta(x, y, t)$ satisfies the differential equation

$$
\frac{\partial \Theta}{\partial t}=\mathcal{D}_{f} \frac{\partial^{2} \Theta}{\partial y^{2}}-\frac{\partial F}{\partial t}
$$

with homogeneous boundary conditions (cf. (3),(5)):

$$
\begin{aligned}
& \left.\frac{\partial \Theta}{\partial y}\right|_{y=l}=0, \\
& \left.\Theta(x, y, t)\right|_{y=0}=0 .
\end{aligned}
$$

By virtue of (5) the initial condition for $\Theta(x, y, t)$ gets the form:

$$
\left.\Theta(x, y, t)\right|_{t=0}= \begin{cases}0 & y=0 \\ -\psi(x) & y>0\end{cases}
$$

The solution of the corresponding homogeneous system $(7)-(9)$ we represent as

$$
\Theta(x, y, t)=Y(y) T(x, t)
$$

Then the corresponding eigenvalues and normalized eigenfunctions for this problem are:

$$
\begin{aligned}
& \chi_{n}=\left(\frac{\pi(2 n+1)}{2 l}\right)^{2}, \quad n=0,1,2, \ldots \\
& Y_{n}(y)=\left(\frac{2}{l}\right)^{1 / 2} \sin \frac{\pi(2 n+1)}{2 l} y .
\end{aligned}
$$

Representing the solution of nonhomogeneous differential equation ( 7 ) in the form

$$
\Theta(x, y, t)=\sum_{n=0}^{\infty} U_{n}(x, t) Y_{n}(y)
$$

and expanding the nonhomogeneous term with the help of the complete set of the eigenfunctions (12), we get the equation for $U_{n}(x, t)$ :

$$
\frac{\partial U_{n}}{\partial t}=-\mathcal{D}_{f} \chi_{n} U_{n}-\left(\frac{2}{l}\right)^{1 / 2} \frac{2 l}{\pi(2 n+1)} \frac{\partial F}{\partial t}
$$

with the initial condition:

$$
U_{n}(x, 0)=-\left(\frac{2}{l}\right)^{1 / 2} \frac{2 l}{\pi(2 n+1)} \psi(x), \quad n=0,1, \ldots
$$

Solution of the equation (14) with (15) has the form:

$U_{n}(x, t)=-\left(\frac{2}{l}\right)^{1 / 2} \frac{2 l}{\pi(2 n+1)} \psi(x) \exp \left(-\mathcal{D}_{f} \chi_{n} t\right)-\int_{0}^{t}\left(\frac{2}{l}\right)^{1 / 2} \frac{2 l}{\pi(2 n+1)} \frac{\partial F(c(x, \tau))}{\partial \tau} \exp \left(-\mathcal{D}_{f} \chi_{n}(t-\tau)\right) d \tau$

Substituting the expression (16) into (13) and taking into account (3) and (6) we get: 


$$
\left.\frac{\partial a}{\partial y}\right|_{y=0}=\left(-\frac{2}{l}\right) \psi(x) \sum_{n=0}^{\infty} \exp \left(-\mathcal{D}_{f} \chi_{n} t\right)-\left(\frac{2}{l}\right) \sum_{n=0}^{\infty} \int_{0}^{t} \frac{\partial F(c(\tau, x))}{\partial \tau} \exp \left(-\mathcal{D}_{f} \chi_{n}(t-\tau)\right) d \tau,
$$

where (due to (4),(5)):

$$
\psi(x)= \begin{cases}F(f(x))=F\left(c_{0}\right), & 0 \leq x \leq x_{0} \\ 0, & x>x_{0}\end{cases}
$$

Let us consider the limit $x \gg x_{0}$. Then by the above conditions the first term in the right-hand side is negligible. Let $\mathcal{D}_{f} t \gg l^{2}$ (where $l$ is the film thickness of the sorption medium) and let $x \gg x_{0}$. Then for the next term in (17) we get:

$$
\begin{aligned}
& -\int_{0}^{t} \frac{\partial F(c(\tau, x))}{\partial \tau} \Delta(t-\tau) d \tau \\
& =-\int_{0}^{t} \frac{\partial F(c(t-\xi, x))}{\partial t} \Delta(\xi) d \xi
\end{aligned}
$$

where $\xi=t-\tau, \Delta(\xi)=\frac{2}{l} \sum_{n=0}^{\infty} \exp \left(-\mathcal{D}_{f} \chi_{n} \xi\right)$. Using the fast decay of the function of $\Delta(\xi)$ for $\xi>0$, the expression (18) can be represented in the form of expansion:

$$
\begin{aligned}
& -\int_{0}^{t} \frac{d F}{d c} \frac{\partial c(x, t)}{\partial t} \Delta(\xi) d \xi \\
& +\int_{0}^{t} \frac{d F}{d c} \frac{\partial^{2} c(x, t)}{\partial t^{2}} \xi \Delta(\xi) d \xi+\mathcal{O}\left(\frac{l^{5}}{\mathcal{D}_{f}^{3}}\right) .
\end{aligned}
$$

Consequently the first and the second terms in (19) for $\mathcal{D}_{f} t \gg l^{2}$ are given by:

$$
\begin{aligned}
& -\frac{d F}{d c} \frac{\partial c(x, t)}{\partial t} \int_{0}^{t} \frac{2}{l} \sum_{n=0}^{\infty} \exp \left(-\mathcal{D}_{f} \chi_{n} \xi\right) d \xi \\
& \simeq-\frac{d F}{d c} \frac{\partial c(x, t)}{\partial t} \frac{2}{l} \sum_{n=0}^{\infty}\left(\mathcal{D}_{f} \chi_{n}\right)^{-1}=-\frac{l}{\mathcal{D}_{f}} \frac{d F}{d c} \frac{\partial c(x, t)}{\partial t}
\end{aligned}
$$

and

$$
\begin{aligned}
& \frac{d F}{d c} \frac{\partial^{2} c(x, t)}{\partial t^{2}} \int_{0}^{t} \xi \frac{2}{l} \sum_{n=0}^{\infty} \exp \left(-\mathcal{D}_{f} \chi_{n} \xi\right) d \xi \simeq \\
& \frac{d F}{d c} \frac{\partial^{2} c(x, t)}{\partial t^{2}} \frac{2}{l} \sum_{n=0}^{\infty}\left(\mathcal{D}_{f} \chi_{n}\right)^{-2}=\frac{l^{3}}{3 \mathcal{D}_{f}^{2}} \frac{d F}{d c} \frac{\partial^{2} c(x, t)}{\partial t^{2}} .
\end{aligned}
$$

$$
\left.\frac{\partial a}{\partial y}\right|_{y=0}=-\frac{l}{\mathcal{D}_{f}} \frac{d F}{d c} \frac{\partial c}{\partial t}+\frac{l^{3}}{3 \mathcal{D}_{f}^{2}} \frac{d F}{d c} \frac{\partial^{2} c}{\partial t^{2}} .
$$

Hence, for $\mathcal{D}_{f} t \gg l^{2}$ and $x \gg x_{0}$ the boundary condition (2) for the model (1)-(5) reduces to the equation:

$$
\frac{u \varepsilon}{s \mathcal{D}_{f}} \frac{\partial c}{\partial x}+\left(\frac{\varepsilon}{s \mathcal{D}_{f}}+\frac{l}{\mathcal{D}_{f}} \frac{d F}{d c}\right) \frac{\partial c}{\partial t}=\frac{l^{3}}{3 \mathcal{D}_{f}^{2}} \frac{d F}{d c} \frac{\partial^{2} c}{\partial t^{2}}
$$

which is nothing but the evolution equation for the concentration of sorbing marker.

\section{DISCUSSION: LIMITING CASES}

We showed that the system of partial differential equations (1)-(5) for the sorbing marker $c(x, t)$ and sorbed marker $a(x, y, t)$ can be reduced (in the long-time limit $\mathcal{D}_{f} t \gg l^{2}$ and $x \gg x_{0}$ ) to unique partial differential equation corresponding the evolution of sorbing marker (23). It is the concentration which experimenter measures at the end $x=L \gg x_{0}$ of packed bed. This equation can be solved explicitly for the case of a weakly nonlinear isotherm of sorption known in the gas-liquid dynamics of sorption [1]. To this end we use approximation for the isotherm of sorption (cf. Eq. (5)):

$$
a(x, y=0, t)=F(c(x, t))=k_{1} c(x, t)+k_{2} c^{2}(x, t),
$$

up to quadratic term, where $\left|k_{2}\right| c(x, t) \ll k_{1}$. Then the equation (23) gets the form:

$$
v \frac{\partial c}{\partial x}+(1+\lambda c) \frac{\partial c}{\partial t}=\tau \frac{\partial^{2} c}{\partial t^{2}}
$$

where

$$
v=\frac{u \varepsilon}{\varepsilon+\mu k_{1}}, \lambda=\frac{2 k_{2} \mu}{\varepsilon+\mu k_{1}}, \tau=\frac{\mu k_{1} l^{2}}{3 \mathcal{D}_{f}\left(\varepsilon+\mu k_{1}\right)}, \mu=s l .
$$

Using the change of variables $\xi=t-x / v, \eta=x / v$ and $c^{*}=\lambda c(x, t)$ we get the Burgers' equation for $c^{*}=\lambda c(x, t)$ :

$$
\frac{\partial c^{*}}{\partial \eta}+c^{*} \frac{\partial c^{*}}{\partial \xi}=\tau \frac{\partial^{2} c^{*}}{\partial \xi^{2}} .
$$




\section{G. EROCHENKOVA}

Its solution is well-investigated (see, e.g. [7]).

Therefore, we reduced the two-dimensional intradiffusion Wicke model (1-5) to the Burgers' equation (26).
Below we consider the Cauchy problem for the equation (25) with initial condition with respect to variable $x$ :

$$
c(x=0, t)=\left\{\begin{array}{l}
f(t) \geq 0, \text { if } t \geq 0, \\
f(t)=0, \text { if } t<0,
\end{array} \quad \int_{-\infty}^{+\infty} f(t) d t=M<+\infty .\right.
$$

Then the solution of the equation (25) has the form:

$$
c(x, t)=\frac{v}{\lambda x} \cdot \frac{\int_{-\infty}^{+\infty} d \omega\left(t-\frac{x}{v}-\omega\right) \exp \left[-\frac{\left(t-\frac{x}{v}-\omega\right)^{2}}{4 \tau x / v}\right] \cdot \exp \left[-\frac{1}{2 \tau} \int_{0}^{\omega} d y \lambda f(y)\right]}{\int_{-\infty}^{+\infty} d \omega \exp \left[-\frac{\left(t-\frac{x}{v}-\omega\right)^{2}}{4 \tau x / v}\right] \exp \left[-\frac{1}{2 \tau} \int_{0}^{\omega} d y \lambda f(y)\right]}
$$

For the simplest case

$$
f(t)=c(x=0, t)= \begin{cases}c_{0}, & 0 \leq t \leq t_{0} \\ 0, & t<0 ; t>t_{0}\end{cases}
$$

one gets from (28):

$$
c(x, t)=\frac{c_{0}}{\lambda} \cdot \frac{[\phi(a+d)-\phi(b+d)] \exp c}{[\phi(a+d)-\phi(b+d)] \exp c+[2+\phi(b)] \exp h-\phi(a)},
$$

where $\phi(z)=\frac{2}{\sqrt{\pi}} \int_{0}^{z} e^{-\xi^{2}} d \xi$ and

$$
\begin{aligned}
& a=\frac{v t-x}{\sqrt{4 \tau x}}, \quad c=\frac{-\lambda c_{0}\left(2 x(v t-x)-\lambda c_{0} x^{2}+v t_{0} x\right)}{4 \tau x}, \\
& b=\frac{v t-x-v t_{0}}{\sqrt{4 \tau x}}, \quad d=-\frac{\lambda c_{0} x}{\sqrt{4 \tau x}}, \quad h=-\frac{\lambda c_{0} v t_{0}}{2 \tau} .
\end{aligned}
$$

If we consider the case $t_{0} \rightarrow 0\left(c_{0} t_{0}=M=\right.$ const $)$ we get

$$
c(x, t)=\frac{1}{\lambda} \sqrt{\frac{\tau}{x / v}} \frac{\left(e^{R}-1\right) \exp \left(-\frac{\left(t-\frac{x}{v}\right)^{2}}{4 \tau x / v}\right)}{\sqrt{\pi}+\frac{\sqrt{\pi}}{2}\left(e^{R}-1\right)\left\{1-\phi\left(\frac{t-\frac{x}{v}}{\sqrt{\frac{4 \tau x}{v}}}\right)\right\}},
$$

where $R=\frac{\lambda c_{0} t_{0}}{2 \tau}$ is the parameter of the non-linearity. It is positive for concave isotherm of sorption (the case of $k_{2}>0$ in (24)) and negative for convex isotherm of sorption (the case of $k_{2}<0$ ).

To investigate the form of the time distribution of the sorbing marker (32) for $t \gg l^{2} / \mathcal{D}_{f}$ and for a fixed length $x=L \gg x_{0}$ one should to consider the following two limiting cases.

\section{Case of small parameter of non-linearity $|R| \ll 1$}

In this case we would expect the diffusion in time to dominate over the non-linearity. For $|R| \ll 1$ the denominator in (32) is $\sqrt{\pi}+\mathcal{O}(|R|)$, uniformly in $x, t, \tau$. Hence $c(x, t)$ may be approximated by

$$
\begin{aligned}
c(x, t) & =\frac{1}{\lambda} \sqrt{\frac{\tau}{\pi x / v}} R \exp \left(-\frac{(t-x / v)^{2}}{4 \tau x / v}\right) \\
& =\frac{c_{0} t_{0}}{\sqrt{4 \pi \tau x / v}} \exp \left(-\frac{(t-x / v)^{2}}{4 \tau x / v}\right) .
\end{aligned}
$$

This is the source solution of the "heat" equation, close 
to the Gaussian shape. So the expectation is verified.

\section{Case of large parameter of non-linearity $|R| \gg 1$}

To discuss the behaviour of the solution (32) for large $|R|$ (i. e., when the non-linearity dominates over the diffusion) it is convenient to introduce the similarity vari- able $T=\frac{t-x / v}{\sqrt{2|\lambda| c_{0} t_{0} x / v}}$. Then we analyze the behaviour of $c(T, x)$, when $|R| \gg 1$ for different ranges of $T$.

1). Let us consider the case when the isotherm of sorption is concave, $R>0$.

Writing (32) as

$$
c(T, x)=\sqrt{\frac{2 c_{0} t_{0} v}{\lambda x}} \cdot g(T, R), \quad g(T, R)=\frac{e^{R}-1}{2 \sqrt{R}} \cdot \frac{e^{-T^{2} R}}{\sqrt{\pi}+\left(e^{R}-1\right) \int_{T \sqrt{R}}^{\infty} e^{-\xi^{2} d \xi}}
$$

we discuss the behaviour of $g$ as $R \rightarrow \infty$ (i. e. $\tau \rightarrow 0$ ) for different ranges of $T$. In all cases, $e^{R}-1 \sim e^{R}$ and we may use

$$
g(T, R)=\frac{1}{2 \sqrt{R}} \cdot \frac{e^{R\left(1-T^{2}\right)}}{\sqrt{\pi}+e^{R} \int_{T \sqrt{R}}^{\infty} e^{-\xi^{2}} d \xi}
$$

$$
\left.c(T, x)\right|_{R \gg 1}= \begin{cases}O\left(R^{-1 / 2}\right) & , T<0 \\ \sqrt{\frac{2 c_{0} t_{0} v}{\lambda x}} \cdot \frac{T}{1+2 T \sqrt{\pi R} \exp \left(R\left(T^{2}-1\right)\right)} & , T \geq 0 .\end{cases}
$$

If $0<T<1$, we have from $(35)$

$$
g \sim T, \quad 0<T<1, \quad R \rightarrow \infty
$$

whereas if $T>1, g \rightarrow 0$ as $R \rightarrow \infty$. Thus $g \rightarrow 0$ except in $0<T<1$, and in that range $g \sim T$. In the original variables the result reads:

$$
\left.c(x, t)\right|_{R \rightarrow \infty}= \begin{cases}\frac{v}{\lambda x}(t-x / v) & , 0<\frac{t-x / v}{\sqrt{\left(2 \lambda c_{0} t_{0} x\right) / v}}<1 \\ 0 & , \text { for the other } x, t\end{cases}
$$

This is the appropriate solution of (25) with a shock (i. e., with a jump) at the point $t=x / v+\sqrt{\left(2 \lambda c_{0} t_{0} x\right) / v}$. The concentration $c$ jumps from zero to $\sqrt{\left(2 c_{0} t_{0} v\right) /(\lambda x)}$. The shock is located at $T=1$ and for large but finite $R(37)$ shows a rapid transition from exponentially small values in $T>1$ to $(1 / \lambda) \sqrt{(4 R \tau v) / x}$ in $T<1$. The transition layer is of thickness $\mathcal{O}\left(\mathcal{R}^{-1}\right)$ around $T=1$ (see Fig. 2)

2 ). In the case when the isotherm of sorption is a convex the solution of (25) is (see Fig. 3 ):

$$
\left.c(T, x)\right|_{|R| \gg 1}= \begin{cases}O\left(|R|^{-1 / 2}\right) & T>0 \\ \sqrt{\frac{2 c_{0} t_{0} v}{|\lambda| x}} \cdot \frac{|T|}{1+2|T| \sqrt{\pi|R|} \exp \left(|R|\left(T^{2}-1\right)\right)}, & T \leq 0\end{cases}
$$




\section{G. EROCHENKOVA}

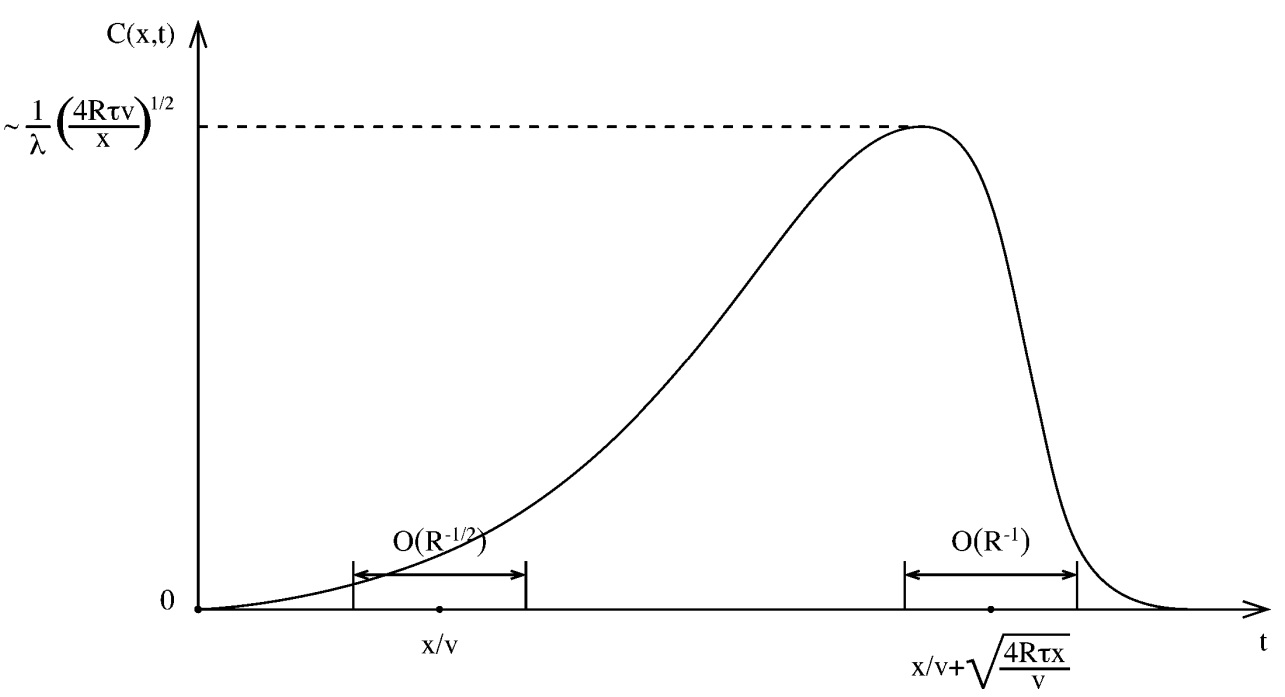

Fig. 2. Shock-wave type solution of Burgers' equation for concave isotherm of sorption.

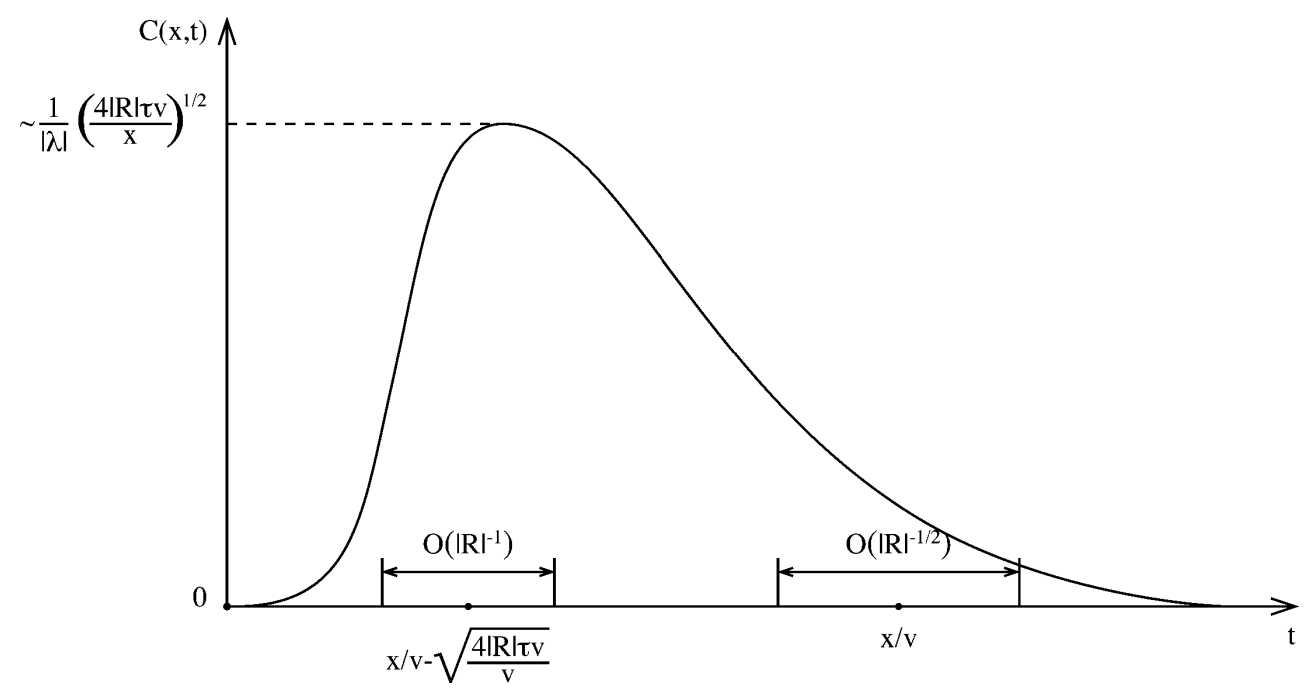

Fig. 3. Shock-wave type solution of Burgers' equation for convex isotherm of sorption.

If $|R| \rightarrow \infty$ ( $x$ is fixed) we get again a solution of the shock-wave type:

$$
\left.c(x, t)\right|_{|R| \rightarrow \infty}= \begin{cases}\frac{v}{|\lambda| x}|t-x / v| & ,-1<\frac{t-x / v}{\sqrt{\left(2|\lambda| c_{0} t_{\mathrm{0}} x\right) / v}}<0 \\ 0 & , \text { for the other } x, t .\end{cases}
$$

\section{CONCLUSION}

- A system of partial differential equations corresponding to two-dimensional intradiffusion (Wicke) model for a weakly nonlinear isotherm of sorption is solved by reducing it to the Burgers' equation. Therefore, in the present paper we solve a model for two-dimensional diffusion. Some limiting cases interested from the physical point of view are considered in details.

- The expression for the concentration (37)-(39) of the sorbing marker for each fixed $x$ has a slanting left slope and a steep right slope in the vicinity of the point $t=x / v+\sqrt{(4 R \tau x) / v}$ in the case of 
concave sorption isotherm (see Fig. 2).

- In this case of (40)-(41) the concentration of the sorbing marker for a fixed $x \gg x_{0}$ has a slanting right slope and the a steep left slope in the vicinity of the point $t=x / v-\sqrt{(4|R| \tau x) / v}$ in the case of convex sorption isoterm (see Fig. 3 ).

- The solutions of (37)-(39) and (40)-(41) have a clear physical interpretation.

- From equation (25) we conclude that the sorbing concentration $c(x, t)$ moves with the velocity $v^{\prime} \sim v /(1+\lambda c)$. The latter means that higher concentrations move slower than the small ones for $\lambda>0$, i. e., $k_{2}>0$. Therefore, the small concentrations arrive to the packedbed output earlier than the high concentrations (see Fig. 2).
- For the case $\lambda<0$ (i. e., $k_{2}<0$ ) higher concentrations arrive to the packed-bed output earlier than small concentrations (see Fig. 3).

- We neglected the non-homogeneity of a sorption medium. In most cases, however, the stationary phase film covering the support surface is nonuniform and fills mainly randomly narrow pores of the support. Latter means that the thickness $l$ of sorption film (see Fig. 1) is stochastic. We return to this problem elsewhere.

\section{ACKNOWLEDGEMENTS}

I would like to thank the referee for useful suggestions.
[1] G. Houghton, J. Phys. Chem. 1, 84 (1963).

[2] P. P. Zolotorev, Zh. Fis. Khim. 48, 1, 113 (1974) (in Russian).

[3] G. V. Yeroshenkova (Erochenkova), S. A. Volkov, K. I. Sakodynsky J. Chromatogr., 198, 4, 377 (1980).

[4] B. Lin, Z. Ma, G. Guiochon, J. Chromatogr. 542, 1, 1 (1991).
[5] V. Wicke, Kolloid Z. 86, 2, 167 (1939).

[6] N. N. Tunitskij, V. A. Kaminskij, S. F. Timashov, The metods of physical chemistry kinetics (Khimiya, Moscow, 1972).

[7] V. Whitham Linear and non-linear waves (New York, 1974).

\title{
РОЗВ’ЯЗОК ДЛЯ МОДЕЛІ ДВОВИМІРНОї ІНТРАДИФУЗІї
}

\author{
Г. Ерошенкова \\ Центр теоретичной фізики, \\ Люміні-Каз 907, Марсель, F-13288, Седекс 9, Франціл \\ galina@cpt.univ-mrs.fr
}

Систему диференціяльних рівнянь у частинних похідних, що відповідає двовимірній моделі інтрадифузії (моделі Віке) для слабо нелінійних ізотерм сорбції, розв'язано способом зведення їі до рівняння Бургерса. У деталях розглянуто деякі граничні випадки, пікаві з точки зору застосування у фізипі. 